\title{
Two-Layered Real-Time Optimization of a Solid Oxide Fuel Cell Stack
}

\author{
Gene A. Bunin, Grégory François, Dominique Bonvin \\ Laboratoire d'Automatique, Ecole Polytechnique Fédérale de Lausanne, \\ CH-1015 Lausanne, Switzerland (e-mail: dominique.bonvin@epfl.ch).
}

\begin{abstract}
The optimal operation of a solid oxide fuel cell stack is addressed in this paper. Real-time optimization, performed at a slow time scale via constraint adaptation, is used to account for uncertainty and degradation effects, while model-predictive control is performed at a faster time scale to reject process disturbances and to safely adapt the system to the specified output constraints following changes in cell power demand. To ensure that these constraints are strictly honored, an adaptation algorithm that uses the built-in constraint handling of quadratic programming is implemented within the model-predictive controller, allowing for the on-line adaptation of the feasibility region as a means to reject uncertainty. Simulation results illustrate the efficacy of this approach in the solid oxide fuel cell system.
\end{abstract}

Keywords: Real-time optimization, Solid oxide fuel cell, Constraint adaptation, Model predictive control, Multilayered optimization.

\section{INTRODUCTION}

With the recent push for alternative energy development, fuel cells have received significant attention from multiple directions. As highly integrated chemical systems, they are subject to a number of safety and physical constraints, all of which must be respected in a real environment. Consequently, feedback control is important, and several control studies have been carried out recently in this area (Golbert and Lewin (2004); Gaynor et al. (2008); Zhang et al. (2008)). On the other hand, research on the optimization of fuel cell processes remains somewhat scarce. Some examples include the work of Tsourapas et al. (2005), where a controller is used to track the calculated optimal values. Zhong et al. (2008) and Chrisovalantou et al. (2009) have both investigated the tracking of the maximum power point, while Golbert and Lewin (2007) have used model-predictive control (MPC) to optimize cell efficiency. Recent experimental studies (Bunin et al. (2010)) have also demonstrated the efficacy of a real-time optimization (RTO) approach for a solid oxide fuel cell (SOFC) stack. Finally, in a direct precursor to this work, Marchetti (2009) has investigated the use of two-layered RTO for an SOFC stack, using both iterative adaptation of the model constraints and on-line constraint control between successive RTO iterations.

Several RTO techniques have been investigated in the literature (Engell (2007)). Only the two-layer methodology is considered in this paper, with the approach of Marchetti (2009) being explored further for the SOFC application. This choice is justified on the grounds that the speed of the system makes the second layer necessary, as a means to supplement the RTO. As was done in the previous work, it is assumed that the optimal operating point is determined by active constraints, which however may change due to degradative effects, process disturbances, or shifts in power demand. Any of these three factors may push the system out of its feasible region, which must be avoided in real applications as it would severely decrease the life of the cell. The goal of the two-layer approach is therefore to first correctly identify the active constraints via the slow-scale optimization layer, and then to make sure that these constraints are enforced via the fast-scale control layer, all while rejecting the effects of potential process disturbances. The effective integration of these two schemes in the context of an SOFC system is the main focus of this contribution.

An important additional problem in SOFC operation is safety during both transient and steady states, as certain constraints may become violated either in transition to a new steady regime, or at the regime itself - the latter corresponding to pure iterative optimization(Bunin et al. (2010)). Prior work by Marchetti (2009) was able to curb the number of constraint violations with the use of MPC, but that work only used soft output constraints in the problem formulation that were ensured via a norm function. Because the SOFC is a system where constraint violations on the order of seconds can have significant detrimental effects on the life of the cell, it is important to minimize all violations - thereby making this approach insufficient in practice. For this purpose, a hard-constraint formulation with a conservative bias initialization, explained via the concept of the feasibility region, is presented in this work.

This paper is organized as follows. Section 2 describes the SOFC process and introduces the key variables. Section 3 formulates the optimization problem, and then reviews the constraint-adaptation mechanism as a means to reject model uncertainty and slow disturbances. The constraint control problem is formulated in Section 4, with a brief review of the soft-constraint MPC approach preceding the proposal for an MPC that uses hard constraints instead. Results from simulation studies using the discussed ap- 
proaches are then given in Section 5. Finally, Section 6 concludes the paper.

\section{THE SOFC SYSTEM}

The diagram of a typical SOFC stack system is presented in Fig. 1. The fuel and air feeds consist of hydrogenwater and nitrogen-oxygen mixtures, respectively, with the electro-oxidation reaction between hydrogen and oxygen producing the electric power in the cell.

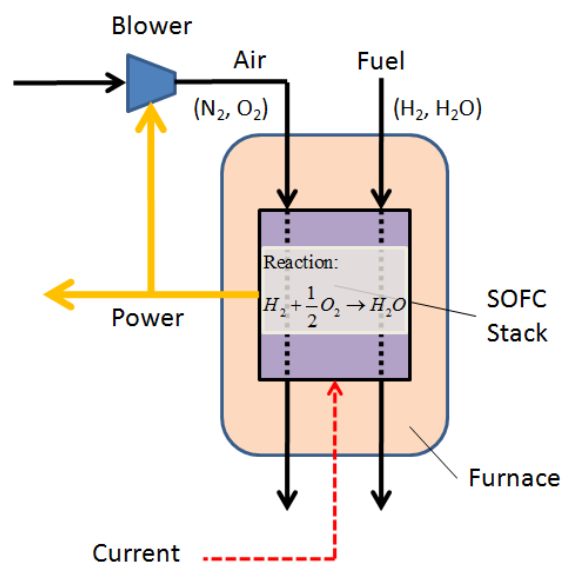

Fig. 1. Diagram of the SOFC stack system.

From a control perspective, the molar flowrates of $H_{2}$ and $\mathrm{O}_{2}$ represent two of the manipulated variables. In most fuel cell applications, the resistance of the load is controlled as well - allowing for accurate manipulation of either the power, voltage, or current. For the specific system used here, the current is chosen as a manipulated variable:

$$
\mathbf{u}=\left[\begin{array}{c}
u_{1} \\
u_{2} \\
u_{3}
\end{array}\right]=\left[\begin{array}{c}
\dot{n}_{H_{2}} \\
\dot{n}_{O_{2}} \\
I
\end{array}\right]
$$

In practice, the set of relevant outputs may be defined as

$$
\mathbf{y}=\left[\begin{array}{c}
y_{1} \\
y_{2} \\
y_{3}
\end{array}\right]=\left[\begin{array}{c}
p_{e l}\left(\dot{n}_{H_{2}}, \dot{n}_{O_{2}}, I\right) \\
U_{\text {cell }}\left(\dot{n}_{H_{2}}, \dot{n}_{O_{2}}, I\right) \\
\nu\left(\dot{n}_{H_{2}}, I\right)
\end{array}\right],
$$

where $p_{e l}$ is the power density (the power divided by the active cell area), $U_{\text {cell }}$ is the cell potential, and $\nu$ is the fuel utilization. It is assumed that a nonlinear, dynamic model is available (Marchetti (2009)).

From a safety standpoint, two of the outputs, $U_{\text {cell }}$ and $\nu$, represent key constraints that must be respected so as to avoid fast system degradation (Gaynor et al. (2008)). Other important constrained quantities include the stack temperature, the air excess ratio $\lambda_{\text {air }}$ (defined as the ratio of $\mathrm{O}_{2}$ fed to the $\mathrm{O}_{2}$ reacted), the fuel flowrate, and the current (Golbert and Lewin (2004); Marchetti (2009)).

The electrical efficiency of the cell stack, which represents the quantity to be maximized, can be defined as the quotient of the total power generated and the amount of fuel/energy consumed:

$$
\eta=\frac{P-P_{\text {blower }}}{\dot{n}_{H_{2}} Q_{L}},
$$

where $P$ is the power output of the stack, $P_{\text {blower }}$ is the portion of the power output that is used to supply the air pump (see Fig. 1), and $Q_{L}$ is the (constant) lower heating value of the fuel.

\section{OPTIMIZATION OF THE SOFC STACK}

\subsection{Formulation of the Optimization Problem}

For the SOFC, the steady-state optimization problem can be written as

$$
\begin{array}{ll}
\max _{\mathbf{u}} & \eta(\mathbf{u}) \\
\text { s.t. } & p_{\text {el }}(\mathbf{u})=p_{\text {el }}^{S} \\
& U_{\text {cell }}(\mathbf{u}) \geq U_{\text {cell }}^{L} \\
& \nu(\mathbf{u}) \leq \nu^{U} \\
& \lambda_{\text {air }}^{L} \leq \lambda_{\text {air }}(\mathbf{u}) \leq \lambda_{\text {air }}^{U} \\
& u_{1} \geq \dot{n}_{H_{2}}^{L},
\end{array}
$$

where the superscript $S$ denotes a desired (setpoint) value, and $L$ and $U$ denote lower and upper bounds, respectively. This formulation is a simplified version from that of Marchetti (2009), with certain constraints dropped as they never become active. The bounds are also chosen as reported, i.e. $U_{\text {cell }}^{L}=0.7 \mathrm{~V}, \nu^{U}=0.7, \lambda_{\text {air }}^{L}=3, \lambda_{\text {air }}^{U}=7$ and $\dot{n}_{H_{2}}^{L}=5 \cdot 10^{-4} \mathrm{~mol} / \mathrm{s}$.

The numerical solution of Problem (4) indicates that the optimum will always lie on a combination of active inequality constraints, one of which will always be either the cell potential or the fuel utilization (Marchetti (2009)).

\subsection{Constraint Adaptation to Combat Uncertainty}

Uncertainty significantly affects both the power density and cell potential, thus making it highly unlikely that the solution of the nominal problem will be optimal, or at times even feasible, for the real SOFC. With the use of online measurements, the effect of model uncertainty may be accounted for via the introduction of a bias in the contraints (Forbes and Marlin (1994); Chachuat et al. (2008)). For Problem (4), the power density and cell potential constraints would then read as:

$$
\begin{aligned}
& p_{e l}(\mathbf{u})+\varepsilon^{p_{e l}}=p_{e l}^{S} \\
& U_{\text {cell }}(\mathbf{u})+\varepsilon^{U_{\text {cell }}} \geq U_{\text {cell }}^{L},
\end{aligned}
$$

where the constraint modifiers $\varepsilon^{p_{e l}}$ and $\varepsilon^{U_{\text {cell }}}$ represent the steady-state bias between the plant and model.

Optimization problem (4) is solved iteratively. Denoting the differences between the plant and predicted values at the $k$ th iteration by $\delta p_{e l, k}$ and $\delta U_{c e l l, k}$, i.e.:

$$
\begin{aligned}
& \delta p_{e l, k}=p_{e l, p, k}-p_{e l}\left(\mathbf{u}_{k}\right) \\
& \delta U_{c e l l, k}=U_{c e l l, p, k}-U_{c e l l}\left(\mathbf{u}_{k}\right),
\end{aligned}
$$

with the subscript $p$ used to represent the measured plant values, one could simply define the modifiers as:

$$
\begin{aligned}
\varepsilon_{k}^{p_{e l}} & =\delta p_{e l, k} \\
\varepsilon_{k}^{U_{c e l l}} & =\delta U_{c e l l, k} .
\end{aligned}
$$




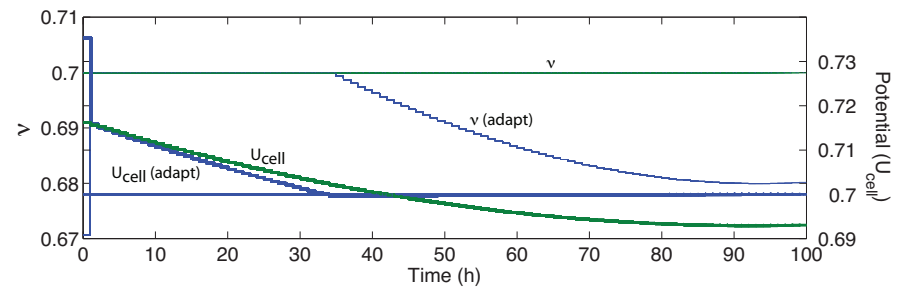

Fig. 2. Effect of process drift on optimization results with and without constraint adaptation. Adaptation is done every hour, with the filter values $K_{p_{e l}}=$ $1, K_{U_{\text {cell }}}=0.7$. Without adaptation, $U_{\text {cell }}$ violates the constraint after about $40 \mathrm{~h}$.

However, the use of a first-order exponential filter to update the modifiers from iteration to iteration is recommended to enforce convergence (Chachuat et al. (2008)):

$$
\begin{aligned}
\varepsilon_{k}^{p_{e l}} & =\left(1-K_{p_{e l}}\right) \varepsilon_{k-1}^{p_{e l}}+K_{p_{e l}} \delta p_{e l, k} \\
\varepsilon_{k}^{U_{c e l l}} & =\left(1-K_{U_{\text {cell }}}\right) \varepsilon_{k-1}^{U_{c e l l}}+K_{U_{c e l l}} \delta U_{c e l l, k},
\end{aligned}
$$

where the filter parameters, $K_{p_{e l}}$ and $K_{U_{\text {cell }}}$, take values between 0 and 1 ( 1 - full adaptation, 0 - no adaptation).

Using the appropriate numerical values for the bounds, the solution of the optimization problem with constraint adaptation is given as follows:

$$
\begin{array}{ll}
\mathbf{u}_{k}^{*}= & \arg \max _{\mathbf{u}} \eta(\mathbf{u}) \\
\text { s.t. } & p_{\text {el }}(\mathbf{u})+\varepsilon_{k-1}^{p_{e l}}=p_{\text {el }}^{S} \\
& U_{\text {cell }}(\mathbf{u})+\varepsilon_{k-1}^{U_{\text {cell }}} \geq 0.7 \mathrm{~V} \\
& \nu(\mathbf{u}) \leq 0.7 \\
& 3 \leq \lambda_{\text {air }}(\mathbf{u}) \leq 7 \\
& u_{1} \geq 5 \cdot 10^{-4} \mathrm{~mol} / \mathrm{s.} .
\end{array}
$$

This iterative algorithm converges to the plant constraints despite plant-model mismatch (Chachuat et al. (2008)), something which has been demonstrated for the SOFC in both simulation (Marchetti (2009)) and experiments (Bunin et al. (2010)). As an aside, if degradation of the metallic interconnector of the SOFC is considered (Brylewski et al. (2001)), it can be seen that a lack of adaptation leads to severe constraint violation in the cell potential over time (Fig. 2).

\section{CONSTRAINT CONTROL}

Much work has been done to investigate the safe transition of cell operating conditions in the case of changing power demand (Golbert and Lewin (2004); Gaynor et al. (2008)), as this transient represents the period of operation when the system is most at risk of violating the safety constraints. Here, the issue is addressed in the context of the proposed two-layer RTO+MPC approach. Since constraint violations that last seconds, rather than minutes, can damage the SOFC, priority is placed on avoiding violations entirely, but not to such an extent that the efficiency of the system is detrimentally reduced.

\section{$4.1 M P C$ with Soft Output Constraints (SCC)}

Although RTO with modifier adaptation is an effective means of converging to the proper set of active constraints,

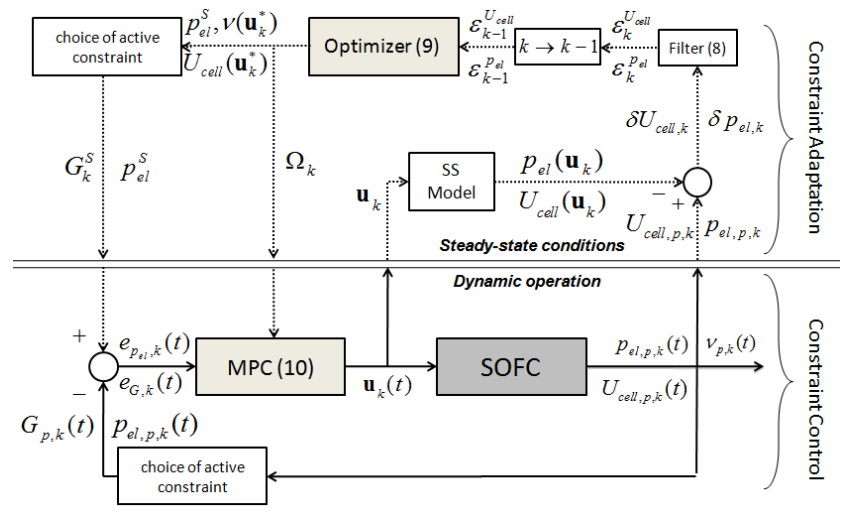

Fig. 3. Two-layer optimization scheme for the SOFC. Constraint adaptation is repeated for the process at steady state and is characterized by the iteration counter $k$, whereas constraint control is done in real time $t . \Omega_{k}$ represents a set of elements supplied by the optimizer $\left(\mathbf{u}_{k}^{*}, w_{U_{\text {cell }}, k}, w_{\nu, k}\right.$ and $\left.\lambda_{\text {air }, k}^{*}\right)$ and required by the MPC, while $G_{k}$ denotes the constraint that is active at the $k$ th iteration (either $U_{\text {cell }}\left(\mathbf{u}_{k}^{*}\right)$ or $\nu\left(\mathbf{u}_{k}^{*}\right)$ ). $e$ are the errors between the constraint setpoints and actual plant values.

it runs the risk of passing through infeasible regions, a case that is exacerbated by the fact that, if a violation occurs, one must wait until the next RTO iteration for corrective measures to be taken. Marchetti (2009) has shown that this problem can be mitigated through the use of an MPC controller that enforces the active plant constraints. The two-layer constraint adaptation and constraint control is presented schematically in Fig. 3.

For constraint control, MPC calculates an optimal control trajectory by minimizing the objective function $J:^{1}$

$$
\begin{aligned}
J(\Delta \mathbf{u}(t)) & =\sum_{l=1}^{n_{p}}\left\{\left\|w_{p_{e l}}\left[p_{e l}(t+l \mid t)-p_{e l}^{S}\right]\right\|^{2}\right. \\
& +\left\|w_{U_{\text {cell }}, k}\left[U_{\text {cell }}(t+l \mid t)-0.7\right]\right\|^{2} \\
& +\left\|w_{\nu, k}[\nu(t+l \mid t)-0.7]\right\|^{2} \\
& +\left\|\mathbf{W}_{\Delta u} \Delta \mathbf{u}(t+l-1)\right\|^{2} \\
& \left.+\left\|w_{\lambda_{\text {air }}}\left[\lambda_{\text {air }}(t+l-1)-\lambda_{\text {air }, k}^{*}\right]\right\|^{2}\right\}
\end{aligned}
$$

where $n_{p}$ denotes the length of the prediction horizon, $w$ the weights, and $\Delta \mathbf{u}$ the vector of input changes, i.e. the control actions. In the SOFC system considered here, most often only one of the inequality constraints (either the potential or the fuel utilization) is active at the optimum, which leaves an extra degree of freedom to track $\lambda_{\text {air }, k}^{*}$, the optimal air excess ratio determined at the $k$ th RTO iteration. As such, either $w_{\nu, k}$ or $w_{U_{c e l l}, k}$ is accordingly set to 0 , depending on the active set. A dynamic matrix of step response coefficients, $\mathbf{B}$, is used to predict the future outputs in the MPC formulation:

$$
\mathbf{y}(t)=\mathbf{y}_{O L}(t)+\mathbf{B} \Delta \mathbf{u}(t)
$$

with $\mathbf{y}_{O L}(t)$ used to define the outputs in the absence of control action (open-loop). The standard way of implementing (10) is to solve it as a quadratic programming

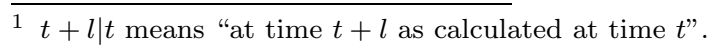


(QP) problem, and apply only the first step of the given trajectory before repeating the process at the next time instant (Qin and Badgwell (2003)). The constraints on the cell potential and fuel utilization are enforced implicitly via the norm functions in (10), and are treated as "soft constraints".

\subsection{MPC with Hard Output Constraints (HCC)}

The SCC's approach to constraint handling becomes its major drawback, as the main priority of this MPC implementation is to get the constrained values of the plant to their desired values, but there still exists the possibility of constraint violations, even if there isn't any plantmodel mismatch. Oscillatory convergence, for example, would induce violations approximately $50 \%$ of the time, the magnitude of which would vary with controller tuning.

For these reasons, an HCC formulation is proposed as a solution, with the principles of constraint adaptation and modifier filtering previously used at the optimization level now being extended to the MPC layer. The idea behind the HCC formulation is, essentially, to exploit the built-in constraint handling of the QP algorithm to satisfy the output constraints of the fuel cell system, while still including some sort of filtering mechanism to guard against uncertainty.

Minimization of the objective function (10) gives:

$$
\begin{aligned}
& \Delta \mathbf{u}^{*}(t)=\arg \min _{\Delta \mathbf{u}} J(\Delta \mathbf{u}) \\
& \text { s.t. } \quad \mathbf{A} \Delta \mathbf{u} \leq \mathbf{b},
\end{aligned}
$$

and is qualified by a linear set of constraints on $\Delta \mathbf{u}$ defined generically here via the matrix $\mathbf{A}$ and the vector $\mathbf{b}$. These linear constraints usually represent the physical bounds on the inputs, but may also be extended to include other variables (Golbert and Lewin (2007)). Because these constraints are written as inequalities, as opposed to norms, they will always be honored by the solution as they are handled explicitly. It is possible to take advantage of this fact by writing the safety constraints in a form recognizable by the QP as well, i.e. as a linear superposition of $\Delta u_{1}, \Delta u_{2}$, and $\Delta u_{3}$.

For fuel utilization, this is relatively straightforward. It is defined as the fraction of the hydrogen that reacts, and may be written as a linear constraint without any modifications:

$$
\nu=\frac{\dot{n}_{H_{2}, \mathrm{reac}}}{\dot{n}_{H_{2}}}=C \frac{u_{3}}{u_{1}} \leq 0.7 \Leftrightarrow-0.7 u_{1}+C u_{3} \leq 0,
$$

from which the formulation in $\Delta u$ terms is trivial and does not change the linear structure of the constraint. The constant parameter $C$ results from Faraday's law (Marchetti (2009)) and is certain, which implies that (13) is a linear combination of the inputs with no parametric uncertainty. Hence, the fuel utilization constraint will always be met if it is included in the QP problem, regardless of the weight tuning that is used.

For the cell potential, the problem is more difficult due to the presence of parametric uncertainty and the fact that the expression for the potential is nonlinear. It is, however, possible to linearize the model around the current operating point at $\tau$ and then add the cell potential modifier $\varepsilon_{\tau}^{U_{c} \text { cell }}$ to offset the uncertainty: ${ }^{2}$

$$
y_{2, \tau}^{l i n}(\mathbf{u})+\varepsilon_{\tau}^{U_{c e l l}} \geq 0.7,
$$

where $y_{2, \tau}^{\text {lin }}(\mathbf{u})$ represents the linearized output equation that is used to define the cell potential as a linear combination of the inputs. $\varepsilon_{\tau}^{U_{\text {cell }}}$ is updated at every MPC time instant $\tau$, using the filter value $\kappa_{U_{c e l l}}$, and achieves the same value as the RTO modifier $\varepsilon_{k}^{U_{c e l l}}$ at convergence for a given set of operating conditions. The cell potential constraint (14) can thus be incorporated into (12).

Putting an explicit, linear bound on the potential introduces an additional constraint to (12) and reduces the area of the feasibility region (FR). At instances when this constraint is active, the MPC will seek to go to this boundary to achieve optimality, and the introduction of a barrier to keep it from going too far seems to be a reasonable choice. The estimation of where this boundary actually lies, however, is inherently inaccurate, since the $\varepsilon_{k}^{U_{c e l l}}$ value itself varies with the operating point. Applying a linear estimation of the constraint and compressing the FR in this manner may thus be detrimental, as the controller may still wander into infeasible regions because of the poor approximation. To avoid this problem, the boundary is artificially pushed in by modifying the initial bias value $\varepsilon_{\tau=1}^{U_{c e l l}}$ whenever a transient state begins, and then allowing this value to be filtered gradually to the new steady-state plant bias from the safe, conservative side. A brief example is given to illustrate this concept.

Example. Consider a two-input problem with two output constraints $y_{a}(\mathbf{u}) \leq 1.25$ and $y_{b}(\mathbf{u}) \geq 0$. As $y_{a}(\mathbf{u})$ is nonlinear and subject to uncertainty, it is linearized, which results in $y_{a}^{\text {lin }}(\mathbf{u})=0.5 u_{1}+u_{2}$, and a modifier $\varepsilon_{\tau}^{a}$ is added to offset the uncertainty. Hence, the input bounds and the constraints for a steady-state operating point $\alpha$ read:

$$
\begin{aligned}
& -1 \leq u_{i} \leq 1, \quad i=1,2 \\
& 0.5 u_{1}+u_{2}+\varepsilon_{\tau}^{a} \leq 1.25 \\
& 2 u_{1}+u_{2} \geq 0
\end{aligned}
$$

The converged feasibility region at this operating point, for which the constraint $y_{b}$ is active, assuming $\varepsilon_{\infty}^{a}=0.25$, is shown in Fig. 4a(i). A change in plant setpoint occurs, and optimality requires that $y_{a}$ becomes active, leading to the definition by the MPC layer, at every MPC instant $\tau$, of a target operating point $\beta_{\tau}$, lying on constraint $y_{a}$. Because the estimation of this constraint with the current modifier is likely to be erroneous, a conservative initialization is applied to the modifier by doubling it (Fig. 4a(ii)). A sufficiently aggressive tuning scheme is then assumed to push the control action to the boundary of the FR at every instant $\tau$. At the next control iteration, the modifier is updated with a filter to bring it closer to the bias of the plant (Fig. 4a(iii)). It should be noted that $y_{a, p}=y_{a}^{\operatorname{lin}}(\mathbf{u})+$ $\varepsilon_{\tau}^{a}$ also moves due to the changes in $\varepsilon_{\tau}^{a}$ along with $\tau$. In Fig. 4a(iv), the algorithm has almost converged, without having violated the constraint.

The strength of this approach may be seen via a comparison to the SCC for the same problem (Fig. 4b(i-iv)).

\footnotetext{
${ }^{2} \tau$ is the discrete time counter for the MPC layer.
} 

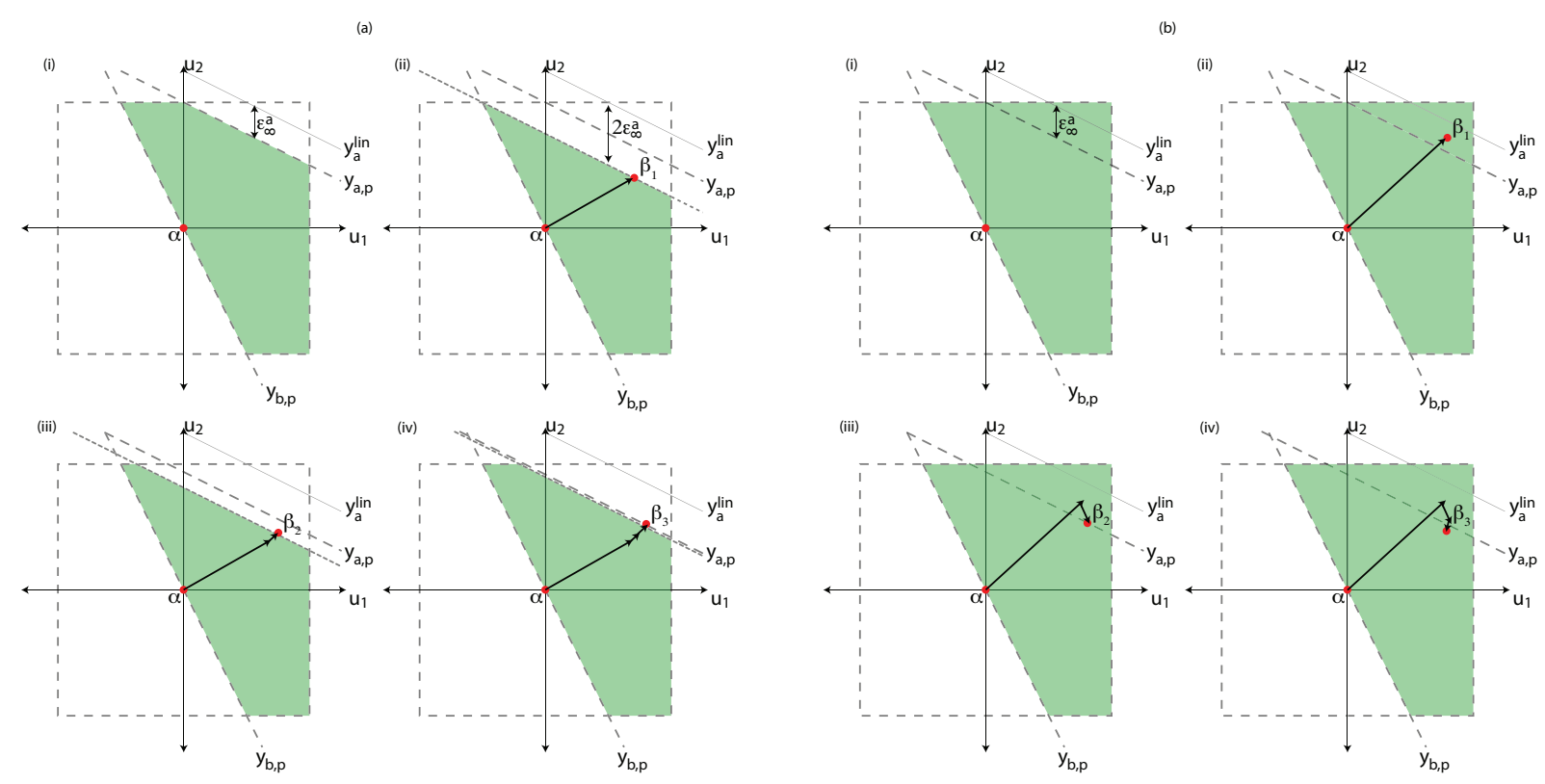

Fig. 4. Theoretical illustration of the HCC (a) and SCC (b) approaches for a problem that involves going from a steady-state operating point $\alpha$ to a new steady-state operating point. The $\mathrm{FR}$ is shaded.

Improper tuning in this case can lead to overshoot and violation of the constraint.

\section{SIMULATION STUDY}

The approach combining RTO and HCC is tested for two scenarios, with the first treating the rejection of fast disturbances and the second looking at the general case of changing power demands (resulting in changing active constraints). Throughout this section, particular attention is given to honoring the cell potential constraint, as it is the one subject to uncertainty, but the inclusion of the fuel utilization boundary in the QP problem is shown as well. For all the simulations, RTO and MPC actions are repeated every $20 \mathrm{~min}$ and $4 \mathrm{~s}$, respectively. Unless otherwise stated, the weights used for the MPC are chosen as $w_{p_{e l}}=10^{-2}, w_{U_{\text {cell }}}=1, w_{\nu}=1.5, w_{\lambda_{a i r}}=10^{3}$, and $\mathbf{W}_{\Delta u}$ a diagonal matrix with elements $\left[10^{-4}, 10,10^{-1}\right]$. This weighing scheme is similar to that of Marchetti (2009), but with both $w_{\lambda_{\text {air }}}$ and $w_{\Delta u_{2}}$ raised to reduce the changes in the air flowrate, as a way to avoid aggressive control action. For the RTO-only results, the filter values $K_{U_{\text {cell }}}=0.7$ and $K_{p_{e l}}=1$ are used.

\subsection{Rejection of Fast Disturbances}

Although RTO alone is capable of dealing with slow disturbances (e.g. the drift in Fig. 2), it cannot satisfactorily deal with disturbances that are on the same time scale as the process dynamics, in which case the control layer is required. This is demonstrated for the case $p_{e l}^{S}=0.4 \mathrm{~W} / \mathrm{cm}^{2}$, where the system has converged to the cell potential constraint (the optimal point). In between RTO iterations, a 10 degree change (1023K to $1013 \mathrm{~K})$ in the fuel inlet temperature occurs and lasts for 2 minutes.

Fig. 5 shows the results for this scenario. The change in temperature leads to an extended violation of the potential constraint in the RTO-only approach since no corrective

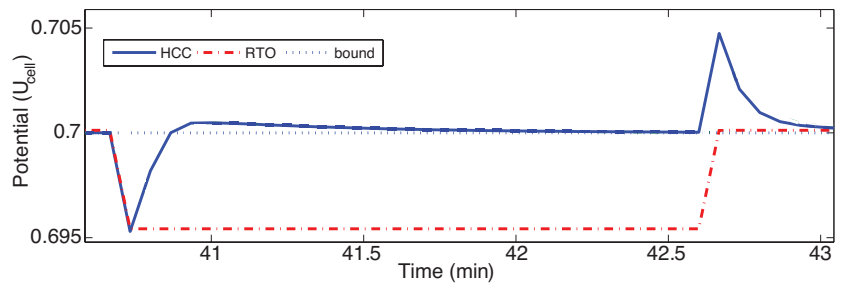

Fig. 5. Rejection of a disturbance in the fuel inlet temperature that occurs in between RTO iterations. Deviations from the constraint bound $U_{\text {cell }}^{L}$ for both the RTO-only and RTO+HCC schemes are shown.

action is taken, but is immediately compensated for in the $\mathrm{RTO}+\mathrm{HCC}$ approach.

\subsection{Changing Power Demand}

In this case, the power density demand acts as a disturbance and varies as follows:

$$
p_{\text {el }}^{S}(t)=\left\{\begin{array}{l}
0.4,0 \leq t<60 \mathrm{~min} \\
0.2,60 \leq t<120 \mathrm{~min} \\
0.3,120 \leq t \leq 180 \mathrm{~min}
\end{array}\right.
$$

The changes in operating conditions cause changes in the set of active constraints, with constraint violation particularly likely at the instances when the cell potential constraint becomes active at time 0 and 120 min. Fig. 6b1,b2 show that these violations are significant for the RTOonly scheme, as it must wait one full iteration (20 min) before a move can be made to leave the infeasible region. In contrast, the situation is handled nicely by the RTO +HCC scheme, which quickly converges to the new constraint.

Fig. 6a2,b2 illustrate the efficacy of the HCC approach in dealing with transients (this is compared to the SCC approach in a2). Following the idea described in Section 4.2, a more conservative linearized constraint (smaller $\mathrm{FR}$ ) is used to compress the input space (Fig. 6b2). The 

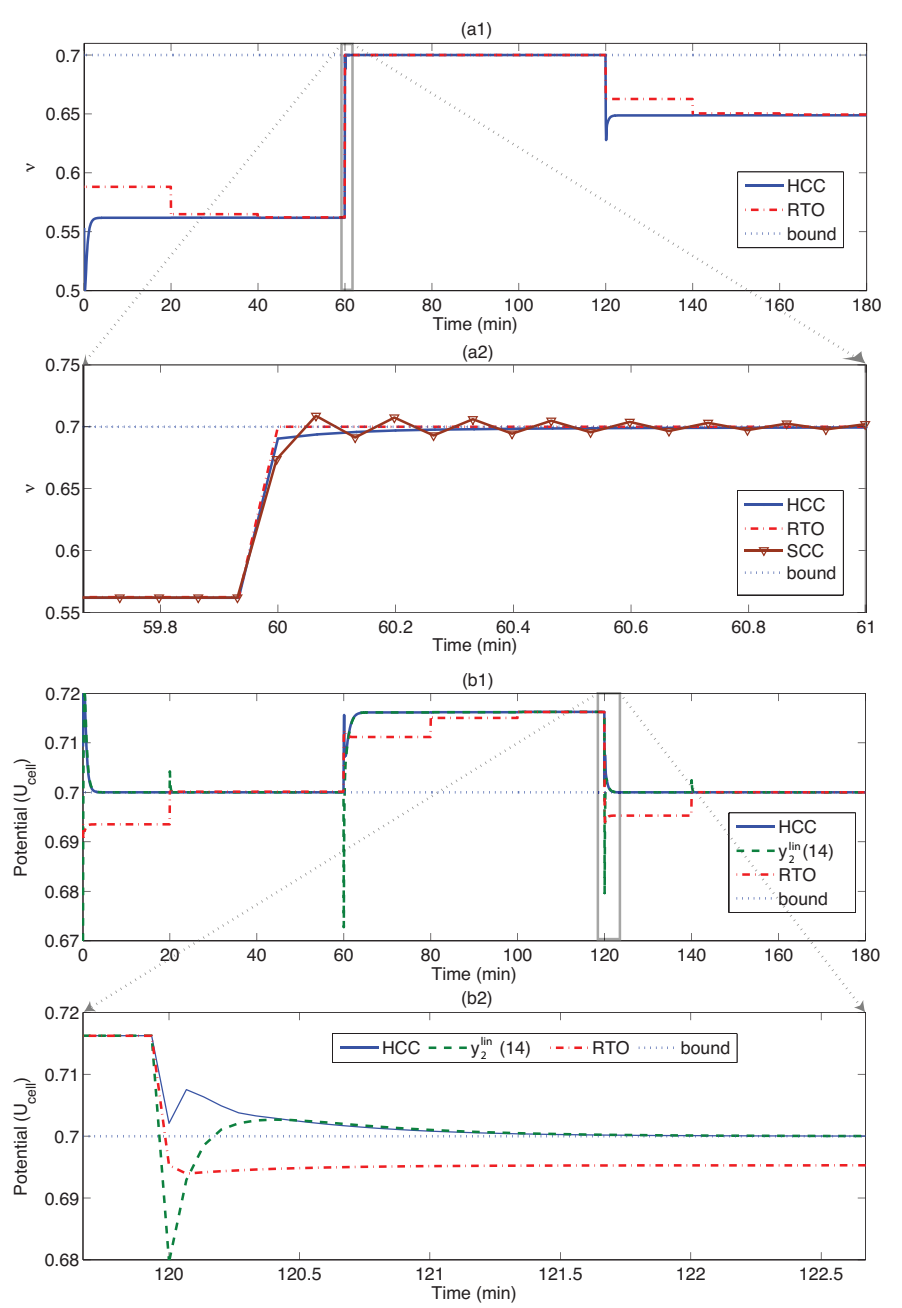

Fig. 6. Performance of the RTO and RTO+MPC schemes in the presence of operating point changes.

corresponding constraint value is then slowly pushed out by $40 \%$ of the offset to the true plant value at each iteration. Upon convergence, the converged feasibility region is achieved. In this case, the filter value $\kappa_{U_{\text {cell }}}=0.4$ leads to a transition time of approximately one minute. An alternate way of looking at this mechanism is to note that the values predicted by the linearized output start out "aggressive" when violation is feared most (thus resulting in more conservative inputs), and then gradually settle down to more realistic estimations ( $y_{2}^{\text {lin }}$ in Fig. 6b2). What results is a somewhat smooth, guided transition to the new operating point. The drawback of MPC with SCC alone is made clear in Fig. 6a2, where it is shown that the SCC approach results in oscillatory convergence under the same set of weights.

\section{CONCLUSIONS}

The results presented in Section 5 illustrate the efficacy of the 2-layer approach combining constraint adaptation and constraint control for the SOFC system in simulated cases. The experimental validation of the layered structured is still an open issue, however, and will be included in further research. Of additional interest is the constraint control formulation presented in Section 4.2, as it has been demonstrated to be both effective and significantly easier to tune in this example. Further study of this approach, as well as its strengths and weaknesses when compared to the standard soft constraint formulation, is planned as well. Finally, it is also evident that RTO+HCC converges much quicker and more reliably to new optimal conditions than RTO alone. While this gain in optimality may not be significant for residential applications (long periods with little change in conditions), applications involving frequent power demand changes (e.g. vehicular) would give rise to greater benefits.

\section{ACKNOWLEDGEMENTS}

The authors would like to thank the Industrial Energy Laboratory (LENI) of EPFL for their assistance in providing data and observations from the experimental SOFC.

\section{REFERENCES}

Brylewski, T., Nanko, M., Maruyama, T., and Przybylski, K. (2001). Application of Fe-16Cr ferritic alloy to interconnector for a solid oxide fuel cell. Solid State Ionics, 143, 131-150.

Bunin, G., Wuillemin, Z., Francois, G., Nakajo, A., Tsikonis, L., and Bonvin, D. (2010). Experimental RTO of an SOFC stack via constraint adaptation. In $23 r d$ ECOS Conference.

Chachuat, B., Marchetti, A., and Bonvin, D. (2008). Process optimization via constraints adaptation. $J$. Process Contr., 18, 244-257.

Chrisovalantou, Z., Papadopoulou, S., Seferlis, P., and Voutetakis, S. (2009). An efficient multi-objective model predictive control framework of a pem fuel cell. In ADCHEM, Istanbul.

Engell, S. (2007). Feedback control for optimal process operation. J. Process Contr., 17, 203-219.

Forbes, J.F. and Marlin, T.E. (1994). Model accuracy for economic optimizing controllers: The bias update case. Ind. Eng. Chem. Res., 33, 1919-1929.

Gaynor, R., Mueller, F., Jabbari, F., and Brouwer, J. (2008). On control concepts to prevent fuel starvation in solid oxide fuel cells. J. Power Sources, 180, 330-342.

Golbert, J. and Lewin, D.R. (2004). Model-based control of fuel cells: (1) regulatory control. J. Power Sources, $135,135-151$.

Golbert, J. and Lewin, D.R. (2007). Model-based control of fuel cells: (2) optimal efficiency. J. Power Sources, 173, 298-309.

Marchetti, A. (2009). Modifier-adaptation methodology for real-time optimization (No. 4449). Ph.D. thesis, EPFL.

Qin, S.J. and Badgwell, T.A. (2003). A survey of industrial model predictive control technology. Contr. Eng. Practice, 11, 733-764.

Tsourapas, V., Stefanopoulou, A., and Sun, J. (2005). Dynamics, optimization and control of a fuel cell based combined heat power (CHP) system for shipboard applications. In American Contr. Conf. 3, 1993-1998.

Zhang, X., Chan, S., Ho, H., Li, J., Li, G., and Feng, Z. (2008). Nonlinear model predictive control based on the moving horizon state estimation for the solid oxide fuel cell. Int. J. Hydrogen Energy, 33, 2355-2366.

Zhong, Z.D., Huo, H.B., Zhu, X.J., Cao, G.Y., and Ren, Y. (2008). Adaptive maximum power point tracking control of fuel cell power plants. J. Power Sources, 176, 259-269. 